\title{
UNCONDITIONAL BASES AND BEST APPROXIMATION IN BANACH SPACES
}

\author{
BY J. R. RETHERFORD ${ }^{1}$ AND R. C. JAMES ${ }^{2}$ \\ Communicated by R. C. Buck, July 10, 1968
}

1. Introduction. Let $\left(x_{i}, f_{i}\right)$ be an unconditional basis for a Banach space $E$. Let $\omega$ denote the positive integers and $\Sigma$ the finite subsets of $\omega$. For $\sigma \in \Sigma$ let

$$
L_{\sigma}=\left[x_{i}: i \in \sigma\right] \text { and } L^{\sigma}=\left[x_{i}: i \in \omega \backslash \sigma\right]
$$

where the bracketed expressions denote the closed linear spans of the indicated elements.

If $S_{\sigma}(x)=\sum_{i \in \sigma} f_{i}(x) x_{i}$ and $S^{\sigma}(x)=x-S_{\sigma}(x)$, let

$$
B_{\sigma}(x)=\left\{z_{0} \in L_{\sigma}:\left\|x-z_{0}\right\|=\inf _{z \in L_{\sigma}}\|x-z\|\right\}
$$

and

$$
B^{\sigma}(x)=\left\{z_{0} \in L^{\sigma}:\left\|x-z_{0}\right\|=\inf _{z \in L^{\sigma}}\|x-z\|\right\} .
$$

Answering a question raised by I. Singer [6], [7], it is shown in [4] that it is always possible to introduce an equivalent norm in $E$ so that $B_{\sigma}(x)=\left\{S_{\sigma}(x)\right\}$ and $B^{\sigma}(x)=\left\{S^{\sigma}(x)\right\}$ for each $x \in E$ and $\sigma \in \Sigma$, i.e., in these particular subspaces of finite dimension and finite codimension the "near point mapping" is single-valued, linear and continuous.

In this note we complete the study of unconditional bases and best approximation in Banach spaces, initiated by Singer [6], by showing that $B_{\sigma}(x)=\left\{S_{\sigma}(x)\right\}$ implies also that $B^{\sigma}(x)=\left\{S^{\sigma}(x)\right\}$ and giving an example where the converse fails. The subsequent theorems and remarks show how exacting this example actually is.

These phenomena described above are peculiar to the theory of unconditional bases. Appropriate examples for the ordered case are given in $\S 3$.

2. Orthogonal sequences. Best approximation for the subspaces $L_{\sigma}$ and $L^{\sigma}$ is closely related to the following orthogonality conditions.

(2.1) Definition. A sequence $\left(y_{n}\right)$ in $E$ is

(i) orthogonal provided $\left\|\sum_{i \in \alpha} a_{i} y_{i}\right\| \leqq\left\|\sum_{i \in \beta} a_{i} y_{i}\right\|$ for $\alpha, \beta, \alpha \subset \beta$, $\beta \in \Sigma$ and arbitrary scalars $\left(a_{i}\right)_{i \in \beta}$;

1 Research supported in part by NSF-GP-5854.
2 Research supported in part by NSF-GP-6653. 
(ii) strictly orthogonal provided the inequality of (i) is strict whenever $\sum_{i \in \beta \backslash \alpha}\left|a_{i}\right| \neq 0$;

(iii) coorthogonal provided $\left\|\sum_{i \in \omega \backslash \beta} a_{i} y_{i}\right\| \leqq\left\|\sum_{i \in \omega \backslash \alpha} a_{i} y_{i}\right\|$ for arbitrary $\alpha, \beta, \alpha \subset \beta, \beta \in \Sigma$ and arbitrary scalars $\left(a_{i}\right)$ for which $\sum_{i \in_{\omega} a_{i} x_{i}}$ converges;

(iv) strictly coorthogonal provided the inequality of (iii) is strict whenever $\sum_{i \in \beta \backslash \alpha}\left|a_{i}\right| \neq 0$.

(2.2) Remarks. (a) A sequence $\left(y_{n}\right), y_{n} \neq 0,\left[y_{n}: n \in \omega\right]=E$, satisfying any of (2.1) (i)-(iv) is necessarily an unconditional basis of $E$.

(b) The unit vector basis of $\left(c_{0}\right)$ satisfies (i) and (iii) above but not (ii) and (iv). The unit vector basis of $l^{1}$ satisfies (ii) and (iv).

(c) (i) and (iii) are equivalent, (ii) implies (iii) and (iv) implies (ii).

There is a certain duality for an unconditional basis $\left(x_{n}\right)$ and its sequence of coefficient functionals $\left(f_{n}\right)$.

(2.3) REMARK. The sequence $\left(x_{n}\right)$ is orthogonal only if $\left(f_{n}\right)$ is orthogonal.

The examples of $(2.2)(\mathrm{b})$ show that the strict version of $(2.3)$ is not valid.

The meaning of Definition (2.1) is given by the following theorem.

(2.4) Theorem. Let $\left(x_{i}\right)$ be an unconditional basis for $E$. Then

(a) $\left(x_{i}\right)$ is orthogonal if and only if $\left\{S^{\sigma}(x)\right\} \in B^{\sigma}(x)$ for all $x \in E$ and $\sigma \in \Sigma$;

(b) $\left(x_{i}\right)$ is strictly orthogonal if and only if $\left\{S^{\sigma}(x)\right\}=B^{\sigma}(x)$ for all $x \in E$ and $\sigma \in \Sigma$;

(c) $\left(x_{i}\right)$ is coorthogonal if and only if $\left\{S_{\sigma}(x)\right\} \in B_{\sigma}(x)$ for all $x \in E$ and $\sigma \in \Sigma$;

(d) $\left(x_{i}\right)$ is strictly coorthogonal if and only if $\left\{S_{\sigma}(x)\right\}=B_{\sigma}(x)$ for all $x \in E$ and $\sigma \in \Sigma$.

Proof. We prove (d). This proof includes the proof of (c) and the others are analogous. If $B_{\sigma}(x)=\left\{S_{\sigma}(x)\right\}$ for all $x \in E$ and $\sigma \in \Sigma$, then for $\alpha \subset \beta, \beta \in \Sigma, x=\sum_{i \in \omega} a_{i} x_{i}$ and $\sum_{i \in \mathbb{N}_{\alpha}}\left|a_{i}\right| \neq 0$ we have (since $\left.L_{\alpha} \subset L_{\beta}\right)$

$$
\left\|x-S_{\sigma}(x)\right\| \geqq\left\|x-S_{\beta}(x)\right\| \text {. }
$$

If equality holds then, since $S_{\alpha}(x) \in L_{\beta}$ and $B_{\beta}(x)$ is one-pointed by hypothesis, we must have $S_{\alpha}(x)=S_{\beta}(x)$, contradicting $\sum_{i \in \beta-\alpha}\left|a_{i}\right|$ $\neq 0$.

On the other hand, if $\left(x_{i}\right)$ is strictly coorthogonal, then for any $\sigma \in \Sigma$ and $y=\sum_{i \in \sigma} b_{i} x_{i} \in L_{\sigma}$ and for any $x \in E$, we have

$$
\left\|x-S_{\sigma}(x)\right\|=\left\|\sum_{i \in \omega \backslash \sigma} f_{i}(x) x_{i}\right\| \leqq\left\|\sum_{i \in \omega \backslash \sigma} f_{i}(x) x_{i}+\left(y+S_{\sigma}(x)\right)\right\|
$$


(taking $a_{i}=f_{i}(x)+b_{i}$ for $i \in \sigma, a_{i}=f_{i}(x)$ for $i \notin \sigma$, and applying (2.1) (iii) card $\sigma$ times) and so $S_{\sigma}(x) \in B_{\sigma}(x)$. If there is a $\sigma \in \Sigma$ and $y$ $=\sum_{i \in \sigma} b_{i} x_{i} \neq S_{\sigma}(x)$ such that $y \in B_{\sigma}(x)$ then there is an $i \in \sigma$ such that $f_{i}(x-y) \neq 0$. Thus,

$$
\begin{aligned}
\left\|x-S_{\sigma}(x)\right\| & =\left\|S^{\sigma}(x)\right\|<\left\|S^{\sigma}(x)+\sum_{i \in \sigma} f_{j}(x-y) x_{j}\right\| \\
& =\|x-y\|=\left\|x-S_{\sigma}(x)\right\| .
\end{aligned}
$$

From this contradiction we infer that $\left\{S_{\sigma}(x)\right\}=B_{\sigma}(x)$.

In view of $(2.2)(\mathrm{c}),(2.4)$ and the examples in $\$ 3$ below, the following example is somewhat surprising.

(2.5) Example. Let $E$ have the same members as $\left(c_{0}\right)$ but with norm defined by

$$
\left\|\left(x_{1}, x_{2}, \cdots\right)\right\|=\sup \left|x_{1}\right| n^{-1} 2^{-n}+\sum_{i=2 ; i \neq n}^{\infty}\left|x_{p_{i}}\right| 2^{-i}
$$

where the sup is for all $n \geqq 2$ and all permutations $\left(p_{i}\right)$ of $\omega \backslash\{1\}$. If $|x|$ denotes the usual norm of $\left(c_{0}\right)$ then it is easily seen that $\frac{1}{4}|x|$ $\leqq\|x\| \leqq \frac{3}{8}|x|$ and so $E$ is isomorphic to $\left(c_{0}\right)$.

1. The unit vector basis $\left(e_{i}\right)$ of $E$ is strictly orthogonal. To see this, let $\beta \in \Sigma$ and $\alpha \subset \beta$. The norm of $\sum_{i \in \alpha} a_{i} e_{i}$ is attained for a particular value of $n$ and a particular permutation $\left(p_{i}\right)$. A larger sum must be obtained when this same $n$ and permutation $\left(p_{i}\right)$ are used for $\sum_{i \in \beta} a_{i} e_{i}$ provided $\sum_{i \in \beta \backslash \alpha}\left|\alpha_{i}\right| \neq 0$.

2. $\quad\left\|\sum_{m=1}^{\infty} \frac{1}{m} e_{m}\right\|=\left\|\sum_{m=2}^{\infty} \frac{1}{m} e_{m}\right\|=\sum_{m=2}^{\infty} \frac{1}{m} 2^{-m}$

and so $\left(e_{i}\right)$ is not strictly coorthogonal.

It is clear that

$$
\left\|\sum_{m=1}^{\infty} \frac{1}{m} e_{m}\right\| \geqq\left\|\sum_{m=2}^{\infty} \frac{1}{m} e_{m}\right\| \text {. }
$$

Also,

$$
\left\|\sum_{m=2}^{\infty} \frac{1}{m} e_{m}\right\|=\sup \sum_{m=2 ; m \neq n}^{\infty} \frac{1}{p_{m}} 2^{-m}=\sum_{m=2}^{\infty} \frac{1}{m} 2^{-m} .
$$

Since

$$
\frac{1}{n} 2^{-n}+\sum_{m=2 ; m \neq n}^{\infty} \frac{1}{p_{m}} 2^{-m} \leqq \frac{1}{n} 2^{-n}+\sum_{m=2 ; m \neq n}^{\infty} \frac{1}{m} 2^{-m}=\sum_{m=2}^{\infty} \frac{1}{m} 2^{-m}
$$

the result follows. 
The meaning of this example in terms of $(2.2)(\mathrm{c})$ and (2.4) is immediate.

Since best approximation to finite dimensional subspaces is unique in strictly convex spaces, it follows that in such spaces (2.4)(b) and (d) are equivalent. In particular, for $E$ Hilbert space we easily obtain the results of [7].

It is interesting that any Banach space $E$ with an unconditional basis $\left(x_{n}, f_{n}\right)$ can always be given a strictly convex norm satisfying (2.4)(b) and (d). Indeed, let

$$
|x|=\sup \left\{\sum_{i=1}^{\infty}\left|f\left(x_{i}\right) f_{i}(x)\right|:\|f\| \leqq 1\right\}+\sum_{i=1}^{\infty}\left|f_{i}(x)\right| 2^{-i}
$$

be the norm of [4] satisfying (2.4)(b) and (d). Let $\||x|\|$ be the strictly convex norm of Day [1] given by

$$
\||| x \mid\|=\left[|x|^{2}+\sum_{i=1}^{\infty}\left|f_{i}(x)\right|^{22^{-2 i}}\right]^{1 / 2} .
$$

It is easy to check that \|\|$\cdot\|\|$ also satisfies (2.4)(b) and (d).

3. $K$-and $T$-norms. Of course, the properties studied in $\$ 2$ are properties of both the norm and the given sequence. Singer [6], [7], motivated by the earlier work of Nikol'skir [2], [3], has called a norm with the property that $\left\{S^{\sigma}(x)\right\}=B^{\sigma}(x)$, for all $x \in E$ and $\sigma \in \Sigma$, an $N K$-norm (with respect to $\left(x_{n}\right)$ ) and a norm with the property that $\left\{S_{\sigma}(x)\right\}=B_{o}(x)$ for all $x \in E$ and $\sigma \in \Sigma$, an $N T$-norm. (This notation perhaps needs explaining: the $N$ is for the Roumanian word for unconditional; the $K$ is for the Russian word for canonical; and the $T$ is for Cebyšev!) In this language the results of the preceding section show that an $N T$-norm is always an $N K$-norm and Example (2.5) shows that there exist $N K$-norms which are not $N T$.

If one no longer considers unconditional bases and considers only the sets $\sigma_{n}=\{1,2, \cdots, n\}$ then there are four properties: (strictly) monotone and (strictly) comonotone analogous to those of (2.1). A theorem analogous to (2.4) is then valid for arbitrary bases. More precisely, a basis $\left(x_{n}\right)$ is strictly monotone if and only if $\left\{S^{\sigma_{n}}(x)\right\}$ $=B^{\sigma_{n}}(x)$ and $\left(x_{n}\right)$ is strictly comonotone if and only if $\left\{S_{\sigma_{n}}(x)\right\}=B_{\sigma_{n}}(x)$ for all $x \in E$ and for all $n$. A norm making the former (latter) true has been called by Nikol'skil a $K$-norm ( $T$-norm).

In this general case the concepts are completely different.

(3.1) EXAMPLE. A $K$-norm which is non- $T$.

Let $E=\left\{\left(x_{i}\right) \in\left(c_{0}\right): \sum_{i=1}^{\infty}\left|x_{i}-x_{i+1}\right|<+\infty\right\}$ with $\left\|\left(x_{i}\right)\right\|$ given by 


$$
\left\|\left(x_{i}\right)\right\|=\sum_{i=1}^{\infty}\left|x_{i}-x_{i+1}\right|+\sum_{i=1}^{\infty}\left|x_{i}\right| 2^{-i}
$$

It is easy to check that $E$ is a Banach space (indeed isomorphic to $l^{1}$ ) and that the unit vectors $\left(e_{n}\right)$ form a conditional basis for $E$. It follows from the triangle inequality that $\left(e_{n}\right)$ is strictly monotone with respect to this norm and so $\|\cdot\|$ is a $K$-norm with respect to $\left(e_{n}\right)$.

However, $\left\|e_{2}+e_{8}\right\|=19 / 8$ and $\left\|e_{1}+e_{2}+e_{3}\right\|=15 / 8$ and so the norm is not even comonotone, i.e., $\|\cdot\|$ is non- $T$ with respect to $\left(e_{n}\right)$.

(3.2) Example. A $T$-norm which is non- $K$.

Let $E$ have the same members as $\left(c_{0}\right)$ but with norm defined by

$$
\left\|\left(x_{i}\right)\right\|=\sup _{n \in \omega}\left\{\frac{1}{n} \sum_{i=1}^{n}\left|x_{i}\right|+\sup _{m \geqq n+1}\left|x_{m}\right|\right\} .
$$

If $\left|\left(x_{i}\right)\right|$ denotes the usual norm of $\left(c_{0}\right)$ it is easy to see that

$$
\left|\left(x_{i}\right)\right| \leqq\left\|\left(x_{i}\right)\right\| \leqq 2\left|\left(x_{i}\right)\right|
$$

and so $E$ is isomorphic to $\left(c_{0}\right)$.

It is easy to check that the unit vector basis $\left(e_{n}\right)$ of $E$ is strictly comonotone and so $\|\cdot\|$ is a $T$-norm. (Indeed the above norm is a special case of the general $T$-norm constructed by Nikol'skil [2].) However, $\left\|e_{1}+e_{2}\right\|=\left\|e_{1}+e_{2}+e_{3}\right\|=2$ and so $\|\cdot\|$ is not strictly monotone, i.e., $\|\cdot\|$ is non- $K$ with respect to $\left(e_{n}\right)$.

\section{BiBLIOGRAPHY}

1. M. M. Day, Strict convexity and smoothness of normed spaces, Trans Amer. Math. Soc. 78 (1955), 516-528.

2. V. N. Nikol'skii, Best appraximation and basis in Fréchet spaces, Dokl. Akad. Nauk SSSR 59 (1948), 639-692. (Russian)

3. - Some questions of best approximation in a function space, Ucen. Zap. Kalininsk. Ped. Inst. 16 (1953), 119-160. (Russian)

4. J. R. Retherford, On Cebysev subspaces and unconditional bases in Banach space, Bull. Amer. Math. Soc. 73 (1967), 238-241.

5. - Schauder bases and best approximation, Collect. Math (to appear).

6. I. Singer, On a theorem of I M. Gel'fand, Uspehi Mat. Nauk 17 (1962), no. 1 (103), 169-176. (Russian)

7. - - Baze in spatii Banach III, Stud Cerc. Mat. 15 (1964), 675-679.

Louisiana State University, Baton Rouge, Louisiana 70803 and State University of New York, Albany, New York 12203 